\title{
Suitability of Visible Light Communication for Platooning Applications: an Experimental Study
}

\author{
Bastien Béchadergue ${ }^{1,2}$, Luc Chassagne ${ }^{2}$ and Hongyu Guan ${ }^{2}$
}

\begin{abstract}
Vehicle-to-vehicle (V2V) communication is a core brick of the smart vehicle, especially in highway platooning configurations where it enables the lateral and longitudinal control of the vehicle trajectory. Although very attractive, the radio systems based on the IEEE 802.11p standard that are classically used for vehicular communication suffer from serious performance degradations in dense traffic scenario, especially in terms of transmission latency. Visible light communication (VLC) has thus been proposed as a complementary technology. In this work, a simple VLC system, based on commercial offthe-shelf (COTS) light-emitting diodes (LED) headlamps and taillights, is presented and tested in several configurations in order to evaluate its compatibility with platooning. It is shown that the system provides, at a data rate of $100 \mathrm{kbps}$, a bit error rate (BER) below $10^{-6}$ up to $30 \mathrm{~m}$ using either the front or back lamps. In addition, the transmission latency is evaluated at $4.2 \mathrm{~ms}$ and it is found that a jamming vehicle overtaking the platoon would not cause any performance degradation. These results thus confirm the interest of VLC for platooning.
\end{abstract}

\section{INTRODUCTION}

Automated highway platoons of vehicles are seen as an interesting intermediate step toward full vehicle automation. In platooning, vehicle-to-vehicle (V2V) communication is vital in order to enable lateral and longitudinal control of the vehicle trajectory [1], [2]. This function is classically performed using radio frequency systems based on the IEEE $802.11 p$ technology. Although they have numerous advantages, these systems can experience in dense traffic scenario severe performance degradations, especially in terms of transmission latency [3]. This transmission latency is however critical in platoon configurations and it must be kept as low as possible in order to reduce the response time of the vehicles and thus the $\mathrm{V} 2 \mathrm{~V}$ distance. The main interest of platoon relies indeed in this $\mathrm{V} 2 \mathrm{~V}$ distance reduction, which allows to optimize road capacity while reducing fuel consumption by limiting the air drag. The European Truck Platooning network targets for example a nominal V2V gap of $0.3 \mathrm{~s}$ on highways, which corresponds to around $10 \mathrm{~m}$ at $130 \mathrm{~km} / \mathrm{h}$ [4]. According to the United States Department of Transportation (USDOT), the transmission latency must thus be kept below $20 \mathrm{~ms}$ when transmitting packets of around 400 bits, despite the various interferences coming from other road users [5].

Visible light communication (VLC), which is not as sensitive as radio communication, has thus been proposed as a

\footnotetext{
${ }^{1}$ Bastien Béchadergue is with the Vedecom Institute, 77 rue des Chantiers, 78000, Versailles, France (bastien. bechadergue@vedecom.fr)

${ }^{2}$ Luc Chassagne and Hongyu Guan are with the Laboratoire d'Ingénierie des Systèmes de Versailles (LISV), University of Versailles Saint-Quentin, University of Paris-Saclay, 10-12 avenue de l'Europe, 78140, Vélizy, France (luc.chassagne@uvsq.fr, hongyu.guan@uvsq.fr)
}

complementary technology [6], [7]. Numerous experimental studies on V2V-VLC have been carried out and data rates of several hundreds of kbps over ranges of a few tens of meters have been demonstrated [8], [9], [10]. However, these works are based on headlamps whereas in platooning, the data transmission is particularly crucial from the leading vehicle (LV) to the following vehicles (FV), which involves necessarily the taillights. In addition, the latency and interference issues have barely been tackled so far [11], [12]. Consequently, the goal of this work is to propose and study a $\mathrm{V} 2 \mathrm{~V}$-VLC prototype able to meet the various latency, range and interference-robustness requirements above-mentioned.

Here, a simple VLC system based on commercial off-theshelf (COTS) light-emitting diodes (LED) headlamps and taillights - in both their traffic and stop modes - is detailed. These light sources are modulated with the on-off keying (OOK) scheme, over which Manchester coding is added, so that they can transmit data at $100 \mathrm{kbps}$. At the receiver end, a custom made circuit composed of a photodiode (PD), a transimpedance amplifier (TIA), a bandpass filter, an amplifier and an analog-to-digital converter (ADC) is employed to collect and record the signal received. This set-up is tested in various configurations typical of highway platooning configurations to show that it can enable data transmission over $30 \mathrm{~m}$ with a bit error rate (BER) below $10^{-6}$, in both the following-to-leading (F2L) and leadingto-following (L2F) vehicle link scenario. It is also shown that the transmission latency reaches only $4.2 \mathrm{~ms}$ and that a vehicle overtaking the platoon while transmitting data over the same frequency band does not have a significant impact on these performances.

In Section II, the structure and implementation of our V2V-VLC prototype is detailed. Then, in Section III, the various experiments carried out are described along with the results obtained. Finally, Section IV provides some concluding remarks and relevant works for the future.

\section{SySTEM DESIGN AND IMPLEMENTATION}

\section{A. Headlamps and Taillights Characteristics}

The VLC system we developed is based on COTS automotive LED headlamps and taillights. Each headlamp is based on a Nichia NC4W121A high-power phosphor LED mounted on a heat sink and integrated in an optic module to meet the automotive lighting standards. The current-voltage characteristic of the whole module has already been detailed in [13], along with its beam pattern. It was shown that this beam pattern has a large peak of luminous intensity of almost $17000 \mathrm{~cd}$ that is then quickly decreasing in every 
surrounding direction so that the distribution remains under $4000 \mathrm{~cd}$ in most of the point tested. The semi-angle at half power in the horizontal plane of reference was then found to be $3^{\circ}$. Finally, the $3 \mathrm{db}$ bandwidth of these headlamps was measured using a PDA8A photo-detector of large bandwidth $50 \mathrm{MHz}$ and was equal to $1.4 \mathrm{MHz}$.

A similar characterization can be carried out for our COTS taillights. Here, these light sources are considered in their two main modes: traffic mode and stop mode. They are composed of several red LED, having their peak of emission at $645 \mathrm{~nm}$ and divided in groups on parallel branches. In traffic mode, the nominal operating point in traffic mode is $12 \mathrm{~V} / 170 \mathrm{~mA}$, which gives a luminous intensity in the reference axis of $15 \mathrm{~cd}$. In stop mode, the nominal operating point is $24 \mathrm{~V} / 300 \mathrm{~mA}$, which gives a luminous intensity of $650 \mathrm{~cd}$. The corresponding light distributions are given in Fig. 1. In traffic mode, the pattern is overall not Lambertian. However, in the horizontal plane of reference, the distribution is indeed Lambertian, with a semi-angle at half power of $20^{\circ}$. In stop mode, the whole pattern is Lambertian, but the semiangle at half power is, this time, around $10^{\circ}$ so the light is slightly more focused. Finally, it is found that the available bandwidth in traffic mode is $2 \mathrm{MHz}$ whereas it is around 1.4 $\mathrm{MHz}$ in stop mode.

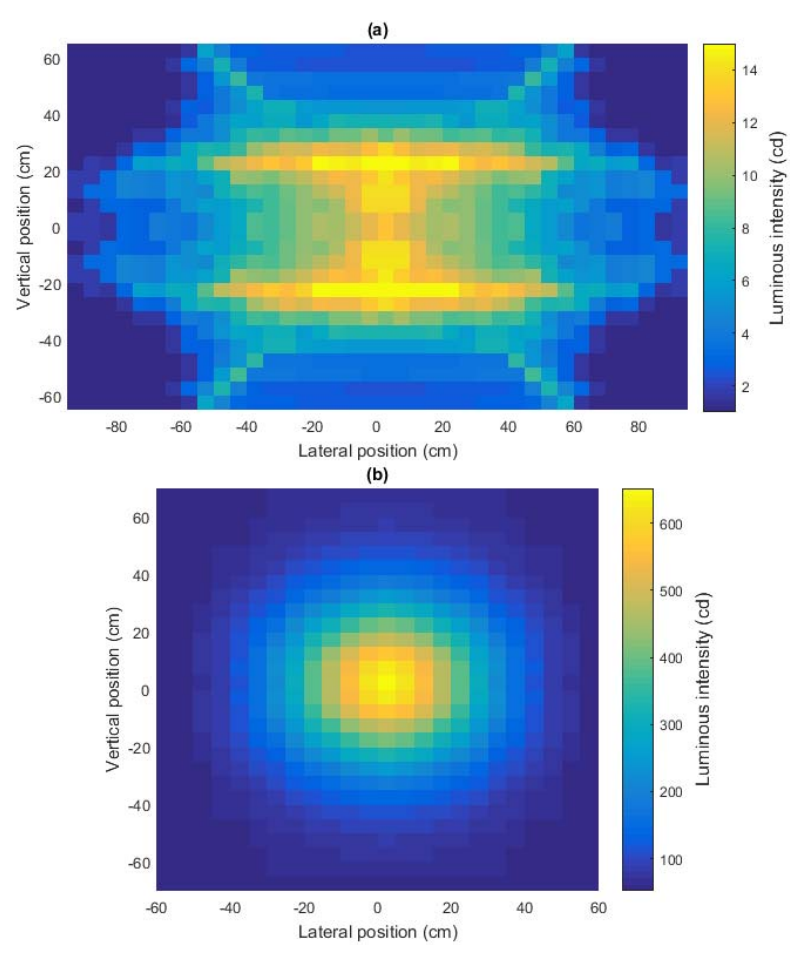

Fig. 1. Spatial distribution of the luminous intensity of a taillight when projected on a vertical plane at $1 \mathrm{~m}$ in (a) traffic mode and (b) stop mode.

\section{B. Receiver Characteristics}

Once the light signal is emitted, and after free space propagation, it must be collected and turned into a workable electrical signal. This is the goal of the front-end stage, which is here custom-made. It is composed of a PD followed by a TIA. The PD, a Hamamatsu S3590, has a large active area of $1 \mathrm{~cm}^{2}$ and a cut-off frequency of around $20 \mathrm{MHz}$ when reverse biased at $15 \mathrm{~V}$. However, this bandwidth is reduced by the inner GBWP of the op-amp on which the TIA is based and by the feedback resistor and capacitance it contains. Here, this op-amp is a LTC6268, of unit GBWP $350 \mathrm{MHz}$, with a feedback resistor that can be varied from 10 to $100 \mathrm{k} \Omega$ and a feedback capacitance of $3.3 \mathrm{pF}$. It is found that the resulting front-end has a $3 \mathrm{db}$ bandwidth of $1.25 \mathrm{MHz}$ when the current-to-voltage gain is kept at 100 $\mathrm{kV} / \mathrm{A}$, whereas its FOV is $55^{\circ}$. These various characteristics are summed up in Table I.

TABLE I

SUMMARY OF THE CHARACTERISTICS OF THE CUSTOM-MADE FRONT-END USED IN THE EXPERIMENTS.

\begin{tabular}{|c|c|}
\hline Parameters & Custom-made front-end \\
\hline Gain & $100 \mathrm{kV} / \mathrm{A}$ \\
\hline Bandwidth & $1.25 \mathrm{MHz}$ \\
\hline Field-of-view & $55^{\circ}$ \\
\hline Active area & $1 \mathrm{~cm}^{2}$ \\
\hline \multirow{2}{*}{ Responsivity } & $0.27 \mathrm{~A} / \mathrm{W} @ 440 \mathrm{~nm}$ \\
\cline { 2 - 2 } & $0.45 \mathrm{~A} / \mathrm{W} @ 645 \mathrm{~nm}$ \\
\hline
\end{tabular}

\section{Prototype Implementation}

These two core components - the transmitting light sources and the front-end receiver - are finally assembled as in Fig. 2 to form our complete VLC prototype.

On the transmitting end, the light intensity of both headlamps in the F2L direction, or both taillights in the L2F direction, is controlled by a MOSFET switch according to the data to transmit. The data signal is first generated with MATLAB and then uploaded to a Tektronix AFG3020 waveform generator, whose output is directly connected to the gate of the driving MOSFET. The data signal is divided into packets, composed first of a header $H=\{1,1,1,1\}$ and then of a data payload of 400 random bits encoded with OOK modulation and Manchester coding. The clock rate $f_{c}$ at which these bits are sent is $200 \mathrm{kHz}$, which means the data rate reached by our system is $100 \mathrm{kbps}$, and that it is compliant with the IEEE 802.15.7 standard [14].

On the receiving end, the light signal is collected by the custom-made front-end previously described and then filtered using a 2nd order analog bandpass filter of high-pass cut-off frequency $1 \mathrm{kHz}$ and low-pass cut-off frequency $100 \mathrm{kHz}$. After filtering, the resulting signal is amplified by a gain made variable with a potentiometer so that the signal peakto-peak amplitude remains at $2 \mathrm{~V}_{p p}$, before digitization with a Tektronix MDO3054 oscilloscope at a sampling rate equal to $12.5 f_{c}$. Fig. 3 shows for example the signal sampled in the case of a F2L data transmission at $30 \mathrm{~m}$.

The signal sampled is finally decoded offline with MATLAB, using the pulse-width decoding method. A first zerocrossing detection step is implemented in order to reconstruct a binary data signal. The number of consecutive samples corresponding to a constant light level is then counted 

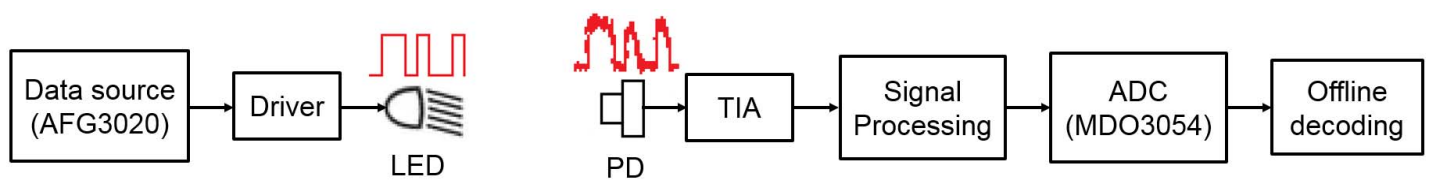

Fig. 2. Emitter and receiver structures used for the VLC experiments.

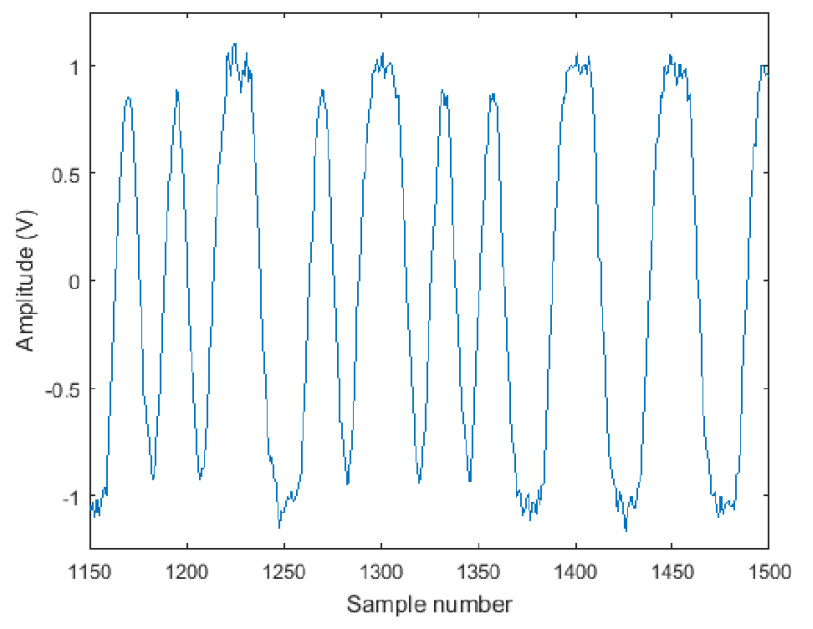

Fig. 3. OOK data signal at $100 \mathrm{kbps}$ digitized in the case of F2L communication at $30 \mathrm{~m}$.

and compared to predefined thresholds to determine the corresponding symbol. An OOK signal coded in Manchester can indeed contain only four distinct symbols: $0,1,00$ and 11. Fig. 4 represents an example of distribution of these count values. The two main peaks on the left represent a single level or two consecutive and identical levels whereas the group around 50 represents the header, the group around 60 is the header preceded or followed by a high level and the group around 70 is the header preceded and followed by a high level. Note that each group is logically centered on a value close to a multiple of 12.5 .

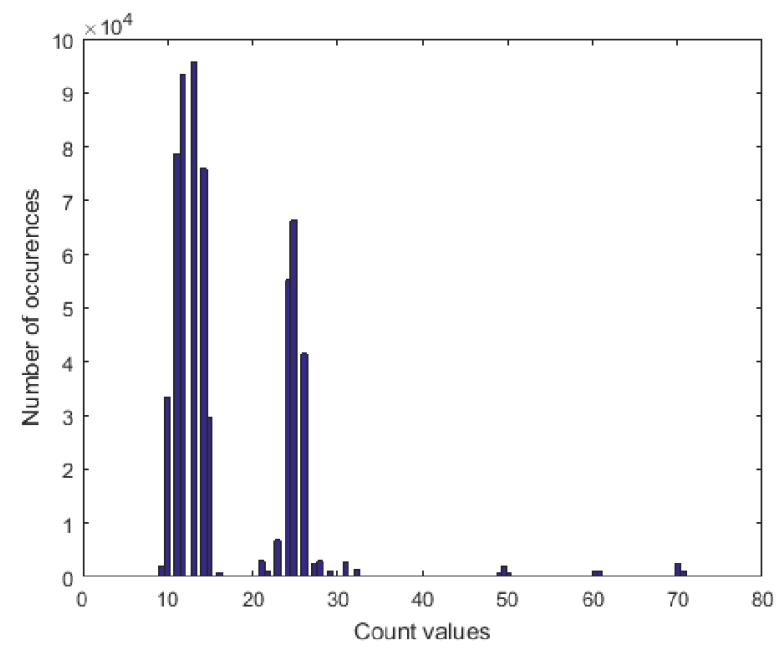

Fig. 4. Distribution of the count values obtained with pulse width decoding in the offline mode, with a sampling rate equal to $12.5 f_{c}$, on packets started by the header $H=1111$.

\section{Performance Investigation of Our Prototype}

\section{A. Experimental Protocol}

In order to verify the suitability of this prototype with platooning, several tests have been carried out with two mobile tables on which are placed the various emission and reception components. On the transmitting side, the two light sources - headlamps or taillights - are fixed with an interdistance of $1.2 \mathrm{~m}, 80 \mathrm{~cm}$ above ground and they emit toward the same direction. On the receiving side, a single VLC receiver is directed toward the headlamps and placed in their horizontal plane of maximum illuminance. The resulting setup is placed in an indoor corridor of length $30 \mathrm{~m}$, illuminated with standard neon tubes driven at $50 \mathrm{~Hz}$ providing a constant average illuminance of 150 lux. Therefore, the perturbations induced are not as massive as they would be in real daytime outdoor applications. These optimal conditions allow us to isolate the impact of the parameters we want to study, like the geometrical configuration or the interferences.

Several geometrical configurations have been tested. As shown by Fig. 5, the relative position of two following vehicles in a platoon can indeed be fully determined by their $\mathrm{V} 2 \mathrm{~V} d$ and the configuration angle $\alpha$ that define the curve radius $R$. Here, two main configurations are tested here: the straight line with varying distance ( $\alpha=0, d$ varying) and the curve at fixed distance ( $d$ fixed, $\alpha$ varying). In any case, the metric used to evaluate the transmission performances is the BER, calculated as the share of wrong bits after decoding over the total number of bits transmitted.

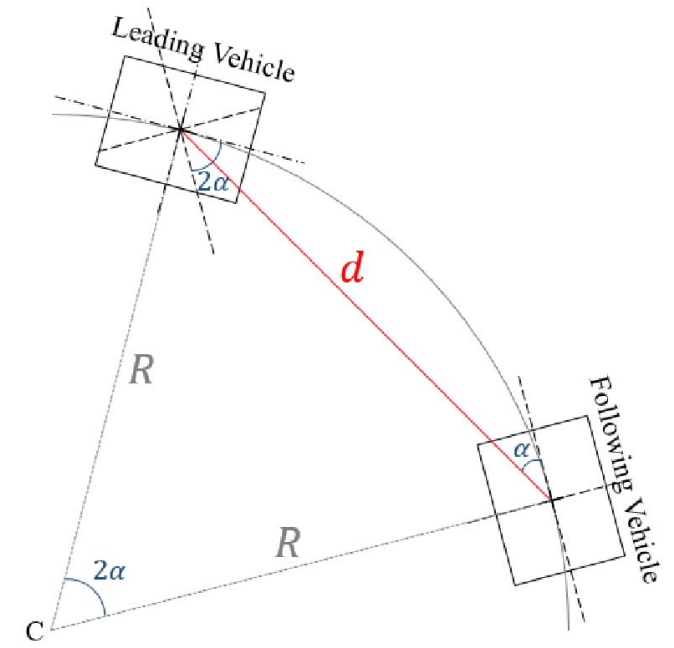

Fig. 5. Two vehicles with an inter-distance $d$, in a curve of center $C$, radius $R$ and fully defined by the angle $\alpha$. 


\section{B. Range and Error Rate Performances}

The performances of the F2L link are first evaluated, in the straight line configuration. The $\mathrm{V} 2 \mathrm{~V}$ distance $d$ is thus varied from $5 \mathrm{~m}$ to $30 \mathrm{~m}$ by steps of $5 \mathrm{~m}$ while the configuration angle is kept null. At each step, $10^{6}$ data bits are sent and the number of wrong bits after decoding is counted in order to evaluate the BER. As shown by Fig. 3, the signal sampled after processing has clear high and low levels, even at $30 \mathrm{~m}$. Consequently, the bits retrieved after decoding do not contain any errors, which means the BER remains below $10^{-6}$ over $30 \mathrm{~m}$.

The curve configuration is then tested. The V2V distance is now set at $10 \mathrm{~m}$, as it could be in a platoon in cruise mode, and the configuration angle is gradually increased up to the value corresponding to a minimal curve radius of $100 \mathrm{~m}$. Note that such a value is not too common in usual highways and is thus a limit case. Despite this fact, the signal received is once again of the form represented on Figure 3 so it can be decoded without errors. In practice, the BER remains once again below $10^{-6}$ for every radius tested.

The headlamps used so far for data transmission are then replaced by the taillights, first in traffic mode and then in stop mode, in order to evaluate the L2F link performances. The V2V distance $d$ is once again varied from $5 \mathrm{~m}$ to $30 \mathrm{~m}$, but this time by steps of $1 \mathrm{~m}$, and the BER is measured at each step. It is found this time that, in traffic mode, there are no decoding errors up to only $10 \mathrm{~m}$. Beyond that distance, the signal reconstructed suffers from too much pulse width distortion. Therefore, as shown by Fig. 6, there is no clear boundary anymore between the group of symbols $0 / 1$ and the group 00/11, which leads eventually to decoding errors. Note, however, that in the curve scenario with $d=10 \mathrm{~m}$, these two groups are still spaced enough to decode all the data transmitted without errors.

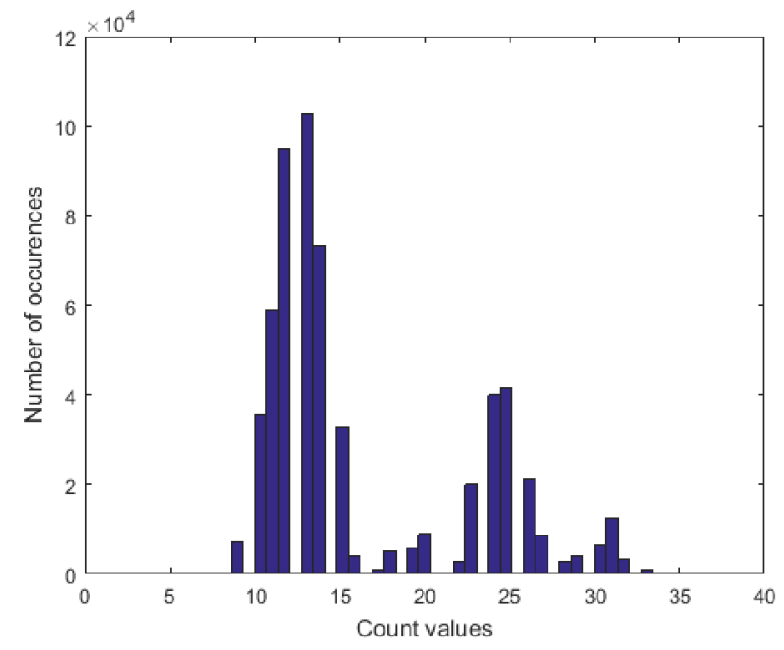

Fig. 6. Distribution of the count values obtained with pulse width decoding with a sampling rate equal to $12.5 f_{c}$, on packets started by the header $H=1111$, when the taillights are used as data transmitter in traffic mode and the V2V distance is $12 \mathrm{~m}$.

In stop mode, the light power available is much larger than in traffic mode, which allows to increase the straight line 'error-free' range of the L2F link. As with the headlamps, this range reaches $30 \mathrm{~m}$ with stop lights, whereas the curve configuration is easily supported. Table II sums up all these results, as well as the results obtained with the headlamps, and shows that bidirectional V2V-VLC communication is supported by our prototype in cruise mode highway platooning at $100 \mathrm{kbps}$ with a BER $<10^{-6}$.

TABLE II

SUMMARY OF THE PERFORMANCES OF OUR VLC PROTOTYPE AT 100 KBPS IN THE VARIOUS CONFIGURATIONS TESTED, FOR A BER $<10^{-6}$.

\begin{tabular}{|c|c|c|}
\hline Link direction & $\begin{array}{c}\text { 'Error-free' } \\
\text { range }\left(\alpha=0^{\circ}\right)\end{array}$ & $\begin{array}{c}\text { 'Error free' } \\
\text { radius }(d=10 \mathbf{m})\end{array}$ \\
\hline F2L & $30 \mathrm{~m}$ & $100 \mathrm{~m}$ \\
\hline L2F (traffic mode) & $10 \mathrm{~m}$ & $100 \mathrm{~m}$ \\
\hline L2F (stop mode) & $30 \mathrm{~m}$ & $100 \mathrm{~m}$ \\
\hline
\end{tabular}

\section{Data Transmission Latency}

If the previous results show that the range of operation of our VLC prototype is compatible with platooning requirements, the transmission latency must also be checked. Here, the transmission latency is defined as the time between the emission of the first bit of a packet and the full reception and decoding of this packet. According to the USDOT, this latency should remain in platooning applications under 20 ms for messages of around 400 bits [5].

In order to verify this criterion, the offline decoding is replaced by an FPGA live decoding algorithm, as reported in [12]. On the emitter side, the driver circuit is connected to another FPGA instead of the waveform generator. This FPGA is programmed to send a packet on demand by pushing a press button whereas the receiving FPGA outputs an enable pulse as soon as the whole packet is received and decoded. Note that here, the packet header is not 1111 as previously but 111100001111 and that it is also added at the end of the message. Figure 7 shows in orange the whole packet taken from the transmitting end and in blue the state of the enable bit, with a peak occurring as soon as the packet reception is finished.

The time between the first rising edge of the packet and the rising edge of this enable bit gives the latency, which is here $4.2 \mathrm{~ms}$ and thus corresponds to the total number of bits transmitted times the transmitting clock period. The first conclusion is that this value is clearly under the 20 ms requirements, which confirms that VLC is an interesting candidate to add redundancy in $\mathrm{V} 2 \mathrm{~V}$ data transmission. It may also be noted that this delay is almost exclusively due to the fact that the transmission of the packet is a serial process. The processing delay induced by the reception and reconstruction stages has indeed been evaluated at $3.6 \mu \mathrm{s}$ and is thus negligible.

The second conclusion is that, contrary to IEEE 802.11p, where the MAC layers are rather heavy and might add excessive delays, the protocol implemented in our system is reduced to a simple header of a few bits. Therefore, most of the available data rate is actually used to transmit the data 


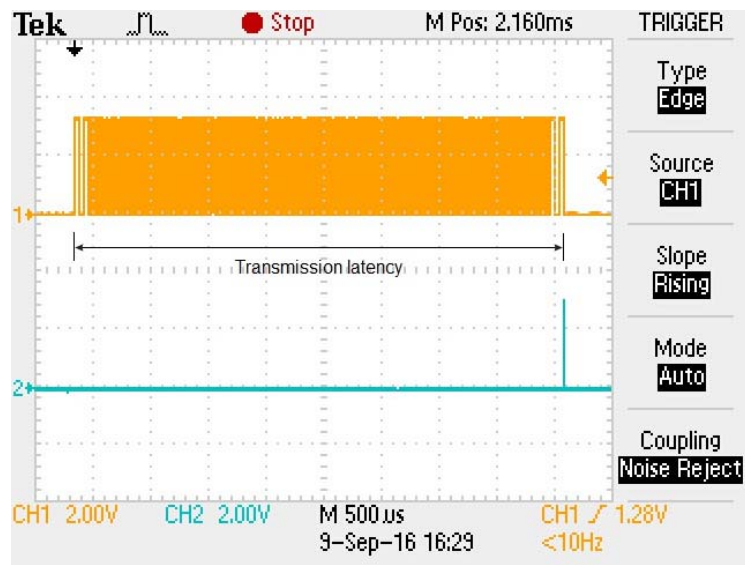

Fig. 7. Transmission latency at $100 \mathrm{kbps}$, measured as the time between the first rising edge of the message (in orange) and the rising edge of the enable bit (in blue).

of interest. In real V2V-VLC communication, the protocols will be probably more complex and thus require a larger share of the available throughput. However, since VLC is a point-to-point communication with rather directive emitters and receivers, the transmission links might experience less interferences, as it is now going to be verified.

\section{Interferences Caused by Other Road Users}

The experiments carried out so far concern only an isolated platoon, where the light detected by each photo-receiver is mainly composed of the light signal of interest plus an additional indoor ambient light. In practice, a platoon is always evolving among other vehicles that are not part of the formation. If these vehicles are also VLC emitters, they might jam the communication links between the members of the platoon. In order to evaluate the strength of these interferences, the set-up described by Figure 8 has been used.

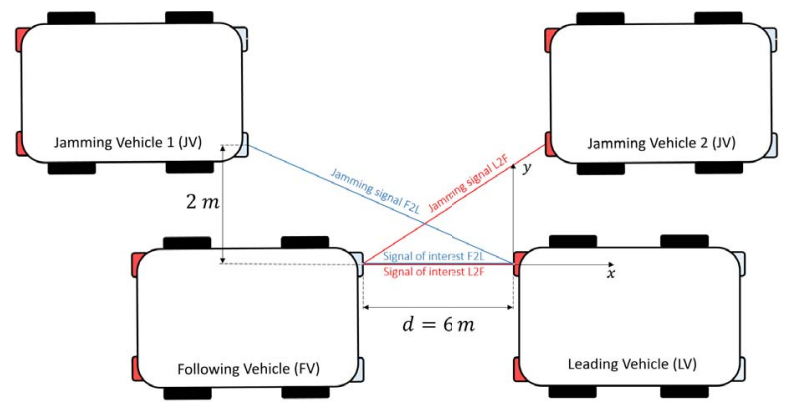

Fig. 8. Set-up used to investigate the impact of the interferences generated by other vehicles, in both the F2L vehicle communication and L2F vehicle communications cases.

A first mobile table serves as receiving vehicle, whereas two other independent tables are used as transmitting vehicles. The first one is placed in the same longitudinal axis as the receiver and represents the vehicle from which we want to receive the data. The second table is parallel with the two others but shifted by $2 \mathrm{~m}$ and represents a jamming vehicle (JV). Both transmitters send different Manchester encoded
OOK signals at a final data rate of $100 \mathrm{kbps}$, without specific additional coding. Note that due to a limited number of headlamps and taillights, only one light is used as transmitter per vehicle. In addition, the distance between the receiving vehicle and the vehicle of interest remains fixed at $6 \mathrm{~m}$ whereas the JV is moving. Consequently, this set-up allows to simulate a vehicle overtaking the platoon, a situation very likely to happen in highway platooning configurations.

In practice, the JV table was moved from $-20 \mathrm{~m}$ to $0 \mathrm{~m}$ by steps of $2 \mathrm{~m}$, with reference to the axis $x$ defined by Figure 8 , with the headlamp as jamming source, and from $-6 \mathrm{~m}$ to $20 \mathrm{~m}$, still by steps of $2 \mathrm{~m}$, with the taillights as jamming source. As summed up in Table III, the JV does not have any impact of the data transmission performances, whatever its distance to the receiver. The light signal of interest remains actually so dominant that the interferences do not degrade the BER at all, in both the L2F and F2L cases.

TABLE III

SUMMARY OF THE PERFORMANCES OF OUR VLC PROTOTYPE AT 100 KBPS IN JAMMING CONFIGURATIONS, WITH A RECEIVER FOV OF $55^{\circ}$.

\begin{tabular}{|c|c|}
\hline Vehicle jammed & BER \\
\hline LV (from $-20 \mathrm{~m}$ to $0 \mathrm{~m}$ along axis $x$ ) & $<10^{-6}$ \\
\hline FV (from $-6 \mathrm{~m}$ to $20 \mathrm{~m}$ along axis $x$ ) & $<10^{-6}$ \\
\hline
\end{tabular}

This behavior can be confirmed by projecting, using the path-loss model [15], the beam patterns of the jamming headlamp or taillight and calculating their contributions to the illuminance at the receiver level. Figure 9 shows the evolution of this share with the longitudinal distance between the JV and the receiver, knowing that the lateral distance is $2 \mathrm{~m}$. In the F2L scenario, the worst case is reached when the distance between the JV and the receiver is $14 \mathrm{~m}$ whereas in the L2F scenario, it is reached around $12.5 \mathrm{~m}$. However, in the first case, the contribution does not exceed $2.75 \%$ whereas in the second case, it is at most $6.5 \%$. These shares are relatively small which explains why the jamming signal cannot be clearly observed in the signal detected. In any case, we can conclude that in a highway platooning configuration, the interferences generated by the other vehicles are not critical and can actually be neglected. Consequently, the communication protocol to use does not need to be too heavy, even though it will probably be more complex than the simple header used so far.

\section{CONCLUSION AND Future WORKS}

This work details the design and implementation of a simple V2V-VLC system based on COTS LED headlamps and taillights on the transmitting end and a custom made receiving end. Several tests are carried out in order to evaluate its performances in terms of range of operation, transmission latency and robustness to interferences. These tests show that it can enable bidirectional data transmission over $30 \mathrm{~m}$ with a BER below $10^{-6}$ between two consecutive vehicles. It is also shown that the transmission latency reaches only $4.2 \mathrm{~ms}$ and that a vehicle overtaking the platoon while transmitting 


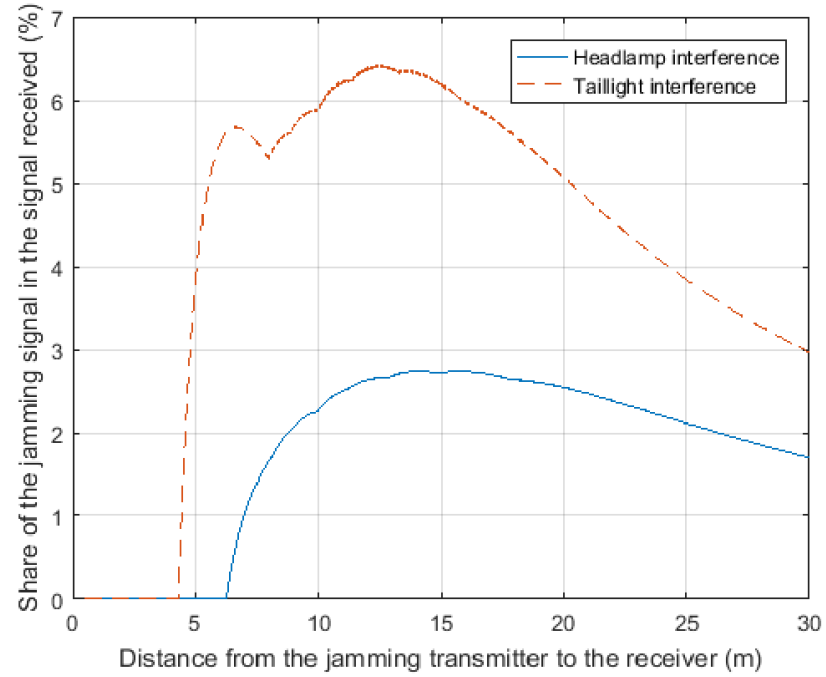

Fig. 9. Evolution with the longitudinal distance of the contribution of the jamming light signal in the total illuminance perceived at the receiver level when the lateral distance is $2 \mathrm{~m}$.

data over the same frequency band does not have a significant impact on these performances. These results thus confirm the suitability of VLC for platooning applications.

However, these indoor tests do not fully take into account the impact of the strong ambient light that would appear in real driving conditions. Therefore, the experiments carried out here must now be reproduced in outdoor conditions, under varying weather conditions like rain of fog in order to investigate the impact of this crucial parameter on the system performances. In addition, the first tests on interferences reported here must be deepened in order to fully define the configurations in which jamming vehicles are an issue.

\section{REFERENCES}

[1] C. Bergenhem, S. Shladover, E. Coelingh, C. Englund, and S. Tsugawa, "Overview of platooning systems", in 19th Intelligent Transportation System (ITS) World Congress, Oct. 2012.

[2] S. Tsugawa, S. Jeschke, and S. E. Shladover, "A Review of Truck Platooning Projects for Energy Savings", IEEE Transactions on Intelligent Vehicles, vol. 1, no. 1, pp. 68-77, Mar. 2016.

[3] A. Böhm, M. Jonsson, and E. Uhlemann, "Performance comparison of a platooning application using the IEEE 802.11p MAC on the control channel and a centralized MAC on a service channel", in 2013 IEEE 9th International Conference on Wireless and Mobile Computing, Networking and Communications (WiMob), Oct. 2013, pp. 545-552.

[4] European Truck Platooning Network, "Vision truck platooning 2025", 2016. [Online] Available: https://www.tno.nl/en/abouttno/news/2016/4/vision-truck-platooning-2025/

[5] "Vehicle Safety Communications Project Task 3 Final Report", United States Department of Transportation, Tech. Rep., Mar. 2005.

[6] M. Abualhoul, M. Marouf, O. Shagdar, and F. Nashashibi, "Platooning control using visible light communications: A feasibility study, in 2013 16th IEEE International Conference on Intelligent Transportation Systems (ITSC), Oct. 2013, pp. 1535-1540.

[7] M. Segata, R. L. Cigno, H. M. M. Tsai, et F. Dressler, "On platooning control using IEEE $802.11 \mathrm{p}$ in conjunction with visible light communications", in 2016 12th IEEE/IFIP Annual Conference on Wireless On-demand Network Systems and Services (WONS), 2016, pp. 1-4.

[8] D.-R. Kim, S.-H. Yang, H.-S. Kim, Y.-H. Son, and S.-K. Han, "Outdoor Visible Light Communication for inter-vehicle communication using Controller Area Network", in 2012 4th International Conference on Communications and Electronics (ICCE), 2012, pp. 31-34.
[9] J.-H. Yoo, R. Lee, J.-K. Oh, H.-W. Seo, J.-Y. Kim, H.-C. Kim, and S.-Y. Jung, "Demonstration of vehicular visible light communication based on LED headlamp", in 2013 5th International Conference on Ubiquitous and Future Networks (ICUFN), Jul. 2013, pp. 465-467.

[10] B. Turan, S. Ucar, S. C. Ergen, and O. Ozkasap, "Dual channel visible light communications for enhanced vehicular connectivity", in 2015 IEEE Vehicular Networking Conference (VNC), Dec. 2015, pp. 84-87.

[11] M. Y. Abualhoul, O. Shagdar, et F. Nashashibi, "Visible Light intervehicle Communication for platooning of autonomous vehicles", in 2016 IEEE Intelligent Vehicles Symposium (IV), 2016, p. 508-513.

[12] B. Béchadergue, L. Chassagne, H. Guan, S. Tohme, and J.-L. Franchineau, "Visible light communication system for platooning applications", in SIA International Conference on Vision, Oct. 2016.

[13] B. Béchadergue, L. Chassagne and H. Guan, "Experimental Comparison of Pulse-Amplitude and Spatial Modulations for Vehicleto-Vehicle Visible Light Communication in Platoon Configurations," Optics Express, vol. 25, no. 20, pp. 24790-24802, Oct. 2017.

[14] S. Rajagopal, R. Roberts, and S.-K. Lim, "IEEE 802.15.7 visible light communication: Modulation schemes and dimming support", IEEE Communications Magazine, vol. 50, no. 3, pp. 7282, Mar. 2012.

[15] J. Kahn and J. Barry, "Wireless infrared communications", Proceedings of the IEEE, vol. 85, no. 2, pp. 265298, Feb. 1997. 